\title{
Inter-laboratory validation of method for determination of rapidly digestible and slowly digestible starch fractions
}

\author{
K. Englyst ${ }^{1}$, A. Goux ${ }^{2}$, A. Meynier ${ }^{2}$, M. Quigley ${ }^{1}$, H. Englyst ${ }^{1}$, O. Brack $^{3}$ and S. Vinoy ${ }^{2}$ \\ ${ }^{1}$ Englyst Carbohydrates Ltd, SO16 7NP UK, ${ }^{2}$ Mondelez International, France and ${ }^{3}$ S. K.I.C, France
}

The rate that starch is digested in the small intestine is a major determinant of the extent that foods raise blood glucose and insulin levels after a meal. This is of nutritional relevance as it is generally recognised that reducing glycaemic responses may have health beneficial effects. Biological origin and processing can influence starch digestibility greatly, and a total starch value alone does not reflect these nutritional properties. A more informative approach has been to divide the available portion into rapidly digestible and slowly digestible starch (RDS and SDS respectively), with an additional resistant starch (RS) fraction reflecting the part escaping digestion in the small intestine. The nutritional relevance of these fractions has been demonstrated previously through a series of in vivo studies $^{(1,2)}$.

The methodology for determination of these starch digestibility fractions was designed to use an excess of pancreatic enzymes for the amylolytic starch hydrolysis, which overcomes variability introduced by enzyme activity or sample amount. Conforming to these principles ensures reproducible measures of; RDS as the glucose release after 20 min incubation adjusted for any free sugars present; SDS as the additional glucose release after $120 \mathrm{~min}$; and RS as the remaining $\operatorname{starch}^{(1)}$.

The present study investigated the performance of the method for RDS and SDS by inter-laboratory trial. Six laboratories took part in the study, two each from UK, France and US. Ten cereal grain products with a range of RDS (23.4-76.9 g/100 g) and SDS (0.8$24.2 \mathrm{~g} / 100 \mathrm{~g}$ ) were tested blind in triplicate, with duplicate analysis of the free sugar glucose and fructose. Analysis was performed in two batches of six samples, with two of the samples included in both batches. Statistical analysis was performed using Jmp $9{ }^{\circledR}$ SAS-Institute to determine the repeatability and reproducibility of the method so that the uncertainty of the RDS and SDS measures could be calculated.

The table shows means and standard deviations for the products analysed in the trial, including the within-laboratory betweenbatch results for the two samples. The repeatability and reproducibility were respectively 0.86 and $1.78 \mathrm{~g} / 100 \mathrm{~g}$ for RDS and 0.72 and $0.88 \mathrm{~g} / 100 \mathrm{~g}$ for SDS. The calculated uncertainty was therefore $3.62 \mathrm{~g} / 100 \mathrm{~g}$ for RDS and $1.91 \mathrm{~g} / 100 \mathrm{~g}$ for SDS, which divided by the median values for each gave global variability of $3 \cdot 21 \%$ for RDS and $2 \cdot 75 \%$ for SDS.

\begin{tabular}{|c|c|c|c|c|}
\hline \multirow[b]{2}{*}{ Sample } & \multicolumn{2}{|c|}{$\underline{\operatorname{RDS}(g / 100 \mathrm{~g})}$} & \multicolumn{2}{|c|}{ SDS $(g / 1 O O g)$} \\
\hline & Mean & $\overline{\mathrm{SD}}$ & Mean & SD \\
\hline Cracker & $66 \cdot 3$ & $1 \cdot 7$ & 4.9 & $1 \cdot 1$ \\
\hline Salt cracker & $55 \cdot 7$ & 1.6 & $7 \cdot 0$ & 0.7 \\
\hline Grains \& chocolate biscuit & $30 \cdot 1$ & 1.4 & $20 \cdot 0$ & $0 \cdot 9$ \\
\hline Whole grain biscuit & $29 \cdot 3$ & $1 \cdot 2$ & $17 \cdot 4$ & $1 \cdot 1$ \\
\hline Rice \& wheat flake cereal & $61 \cdot 2$ & $2 \cdot 0$ & 1.6 & $1 \cdot 3$ \\
\hline Toasted brioche & $57 \cdot 9$ & $1 \cdot 1$ & $0 \cdot 2$ & $0 \cdot 3$ \\
\hline Honey \& chocolate biscuit & $23 \cdot 4$ & $1 \cdot 3$ & $17 \cdot 9$ & $1 \cdot 0$ \\
\hline Butter biscuit & $47 \cdot 6$ & $1 \cdot 0$ & $2 \cdot 7$ & $0 \cdot 5$ \\
\hline
\end{tabular}

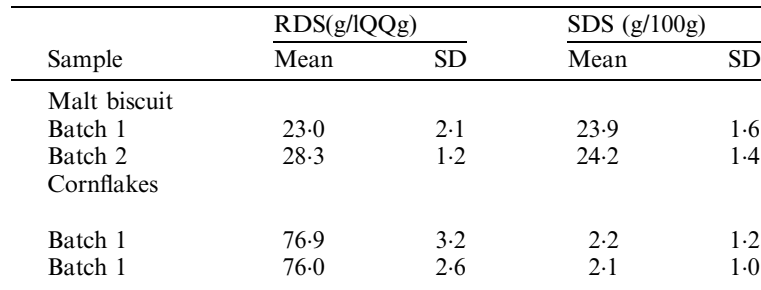

In conclusion, this trial demonstrates satisfactory variability providing validation for the method transfer between laboratories. This lends further support to the applicability of the RDS and SDS measures, including their use in an EFSA claim relating to SDS ${ }^{(3)}$.

1. Englyst KN, Englyst HN, Hudson GJ, et al. (1999) Am J Clin Nutr 69, 448-454.

2. Englyst HN, Kingman SM, Hudson GJ, et al. (1996) Br J Nutr 75, 749-755.

3. EFSA Panel on Dietetic Products, Nutrition and Allergies (2011) EFSA Journal 9, 2292-2307. 